\title{
Study on the synthesis and scale inhibition performance of phosphate free environmental protection type poly epoxy succinic acid
}

\author{
Huang Changshan ${ }^{1, a^{*}}$, Xu Huiwu ${ }^{2, b}$, Chen $\min ^{3, c}$, Zhang $\min ^{4, d}$, Wu Jinying ${ }^{5, e}$ \\ ${ }_{1,2,3,4,5}$ Energy Research Institute of Henan Academy of Sciences Co. Ltd., Zhengzhou, 450008, \\ China \\ a,b,c,d,e13937140170@163.com
}

\begin{abstract}
Keywords: Environmental protection, Poly epoxy succinic acid, Synthesis, Scale inhibition performance.

Abstract. Using maleic anhydride as raw material, sodium tungstate as catalyst, hydrogen peroxide as oxidizing agent, calcium hydroxide as initiator, the synthesis conditions of the best scale inhibition performance of poly epoxy succinic acid were studied by using the method of polymerization: maleic anhydride dosage was $9.8 \mathrm{~g}$, the catalyst sodium tungstate amount used is $0.33 \mathrm{~g}$, the cyclization response $\mathrm{pH}$ value is 4 , the initiator calcium hydroxide joins the quantity is $0.5 \mathrm{~g}$, polymerization temperature $82^{\circ} \mathrm{C}$, polymerization time $2 \mathrm{~h}$. The scale inhibition performance of the poly epoxy succinic acid was evaluated by atomic absorption spectrophotometry: When gathers the poly epoxy succinic acid joins the quantity is $8 \mathrm{mg} / \mathrm{L}$, the best effect of the scale inhibition rate of barium sulfate is $98 \%$; When gathers the poly epoxy succinic acid joins the quantity is $40 \mathrm{mg} / \mathrm{L}$, the best effect of the scale inhibition rate of strontium sulfate is $90.4 \%$.
\end{abstract}

\section{Introduction}

Phosphorus free environment-friendly water treatment agent has been recognized as the development direction of twenty-first Century water treatment agent ${ }^{[1-2]}$. Poly epoxy succinic acid was twentieth Century 90's USA Betz lab first developed a non phosphorus nitrogen free "green" water treatment agent ${ }^{[3]}$, Japan and other countries have begun to polye poxy succinic acid and its derivatives were studied by ${ }^{[4]}$.

Research shows that, poly epoxy succinic acid has good synergistic effect, is a kind of low phosphorus scale inhibitor ${ }^{[5-7]}$ or non phosphorus scale and good effect, is a promising inhibitor. The domestic study on poly epoxy succinic acid in the lab, the production process is not stable, it is difficult to obtain the wide application of ${ }^{[8-10]}$. Therefore, this paper using maleic anhydride as raw material, sodium tungstate as catalyst, hydrogen peroxide as oxidizing agent, calcium hydroxide as initiator, poly epoxy succinic acid by hydrolysis, oxidation, polymerization, ring acidification method, and the influence factors on product performance of scale inhibition was investigated experimentally.

\section{Experiments}

Reagents. Maleic anhydride, 30\% hydrogen peroxide, sodium hydroxide, calcium hydroxide, sodium tungstate, sodium chloride, sodium sulfate, barium chloride, strontium chloride, anhydrous ethanol, hydrochloric acid, all the reagents were analytical pure grade reagent.

Instruments. 501BS type super constant temperature water bath; JB90-D type strong electric mixer; 402 type vacuum drying box; WH-1 type SR hollow cathode lamp; WH-1 type barium hollow cathode lamp; LAB-200 type fully automatic atomic absorption spectrophotometer.

Experimental methods. $9.8 \mathrm{~g}$ of maleic anhydride and $18 \mathrm{ml}$ deionized water were added to the beaker, stirring to dissolve, pour in three mouth flask. Through the constant pressure drop funnel drop add a certain amount of $50 \%$ sodium hydroxide solution, when the bath temperature rose to $60^{\circ} \mathrm{C}$, adds the catalyst sodium tungstate and within 30min uniform droplets with a certain amount of mass fraction as $30 \%$ hydrogen peroxide oxidation agent, keep the temperature of water bath at $60^{\circ} \mathrm{C}$, polymerization reaction $2 \mathrm{~h}$ after, with $50 \%$ sodium hydroxide solution adjusting the $\mathrm{pH}$ value in 4 , 
heating, when the rose to a certain temperature, two batches of the canucks initiator calcium hydroxide, temperature is kept constant, a period of reaction, the final product was poly epoxy succinic acid.

Scale inhibition performance evaluation. According to the oil and gas industry standards SY/T 5673-93 " Anti scaling agent performance evaluation method for oilfield ", scale inhibition performance of synthetic product, scale object for barium sulfate and strontium sulfate.

\section{Results and discussion}

Effect of amount of catalyst on the product scale inhibition rate. Maleic anhydride was $9.8 \mathrm{~g}$, adjust $\mathrm{pH}$ value with $50 \%$ sodium hydroxide solution, hydrolysis $\mathrm{pH}$ was 6 7, cyclization temperature was $60^{\circ} \mathrm{C}$, cyclization $\mathrm{pH}$ was 4 , cyclization time was $2 \mathrm{~h}$, initiator dosage was $0.5 \mathrm{~g}$, polymerization time was $2 \mathrm{~h}$, polymerization temperature was $82^{\circ} \mathrm{C}$, poly epoxy succinic acid dosage was $8 \mathrm{mg} / \mathrm{L}$. Effect of catalyst amount on the product of $\mathrm{BaSO}_{4}$ scale inhibition performance, see Fig.1.

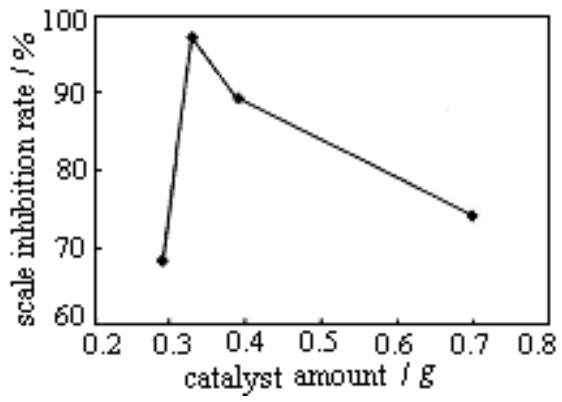

Fig. 1 The influence of catalyst amount on the product scale inhibition rate

The catalyst effect of epoxy succinic acid sodium (or epoxy succinic acid sodium hydrogen) yield, which has a certain relation with the nature of the catalyst, the catalyst concentration is low, the effect is not obvious, the resulting product is mainly epoxy succinic acid sodium salt; catalyst concentration is high, then the by-products of tartaric acid, We can see from Fig.1, when the amount of catalyst was $0.33 \mathrm{~g}$, product has good scale inhibition rate.

Effect of cyclic pH on the scale inhibition rate of the product. Maleic anhydride was $9.8 \mathrm{~g}$, adjust $\mathrm{pH}$ value with $50 \%$ sodium hydroxide solution, hydrolysis $\mathrm{pH}$ was $6 \sim 7$, cyclization temperature was $60^{\circ} \mathrm{C}$, cyclization time was $2 \mathrm{~h}$, catalyst was $0.33 \mathrm{~g}$, initiator dosage was $0.5 \mathrm{~g}$, polymerization time was $2 \mathrm{~h}$, polymerization temperature was $82^{\circ} \mathrm{C}$, poly epoxy succinic acid dosage was $8 \mathrm{mg} / \mathrm{L}$. Cyclization of effect of $\mathrm{pH}$ on the product of $\mathrm{BaSO}_{4}$ scale inhibition performance, see Fig.2.

In acid solution, the generation of intermediate product of epoxy succinate sodium prone to side reaction and hydrogen ion, hydrolysis of sodium tartrate; the increase of $\mathrm{pH}$ solution is alkaline, and can accelerate the decomposition of hydrogen peroxide, to reduce hydrogen peroxide oxidation ability, maleic acid sodium can not fully epoxidation, leading to reduced, the intermediate product of epoxy sodium succinate yield concentration to reduce, reduce the effective content of poly epoxy succinic acid in the end product, weakening the scale inhibition performance of product. We can see from Fig.2, when the ring $\mathrm{pH}$ was 4 , the product has good scale inhibition rate.

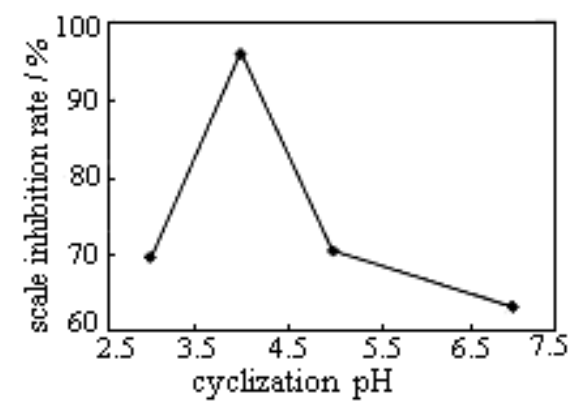

Fig. 2 The influence of cyclization $\mathrm{pH}$ on the product scale inhibition rate 
Effects of initiator concentration on scale inhibition rate of the product. Maleic anhydride was $9.8 \mathrm{~g}$, adjust $\mathrm{pH}$ value with $50 \%$ sodium hydroxide solution, hydrolysis $\mathrm{pH}$ was $6 \sim 7$, cyclization temperature was $60^{\circ} \mathrm{C}$, cyclization time was $2 \mathrm{~h}$, cyclization $\mathrm{pH}$ was 4 , catalyst was $0.33 \mathrm{~g}$, polymerization time was $2 \mathrm{~h}$, polymerization temperature was $82^{\circ} \mathrm{C}$, poly epoxy succinic acid dosage was $8 \mathrm{mg} / \mathrm{L}$, influence of initiator dosage on product resistance $\mathrm{BaSO}_{4}$ scale properties, see Fig.3.

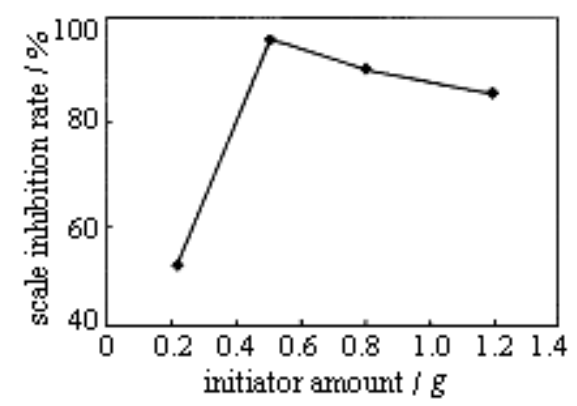

Fig. 3 The influence of initiator amount on the product scale inhibition rate

Initiator dosage has great impact on the degree of polymerization, i.e. that cause a quantity of agent is too small, the synthesis of polyepoxysuccinic acid of high degree of polymerization; initiator dosage too much smaller degree of polymerization, the synthesis of polyepoxysuccinic acid, poly epoxy succinic acid polymerization of sodium is too large or too small, the scale inhibition performance are poor. We can see from Fig.3, initiator dosage was $0.5 \mathrm{~g}$, the product has good scale inhibition rate.

Effect of polymerization temperature on the scale inhibition rate of the product. Maleic anhydride was $9.8 \mathrm{~g}, 50 \%$ sodium hydroxide solution adjust $\mathrm{pH}$, hydrolysis $\mathrm{pH}$ value $6 \sim 7$, cyclization temperature was $60^{\circ} \mathrm{C}$, cyclization time was $2 \mathrm{~h}$, cyclization $\mathrm{pH}$ was 4 , catalyst was $0.33 \mathrm{~g}$, initiator dosage was $0.5 \mathrm{~g}$, polymerization time was $2 \mathrm{~h}$, poly epoxy succinic acid dosage was $8 \mathrm{mg} / \mathrm{L}$. The influence of temperature on the product of $\mathrm{BaSO}_{4}$ scale inhibition performance of polymerization, see Fig.4.

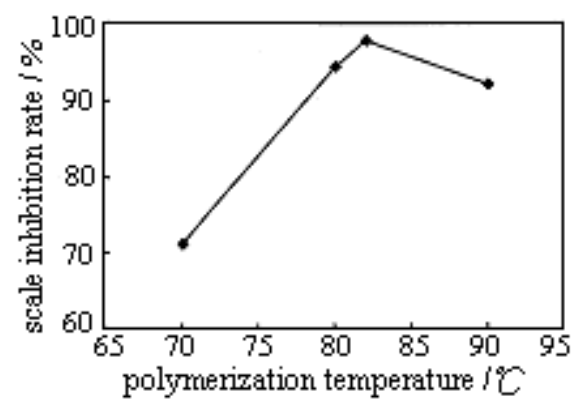

Fig. 4 The influence of polymerization temperature on the product inhibition rate

At low temperature, the polymerization reaction is slower, the strengthening of the hydrolysis reaction of epoxy monomer sodium succinate, monomer polymerization velocity greater than the velocity of hydrolysis; with the increase of temperature, initiator decomposition rate agent to speed up, the degree of polymerization increases, the relative molecular weight of the product increased gradually, the scale inhibition performance to achieve the best; when the temperature is greater than $82^{\circ} \mathrm{C}$ poly epoxy succinic acid sodium, high degree of polymerization, the relative molecular weight of the product may be more than with relative molecular weight of the best scale inhibition performance of scale inhibition effect, resulting in decreased. We can see from Fig.4, the polymerization temperature $82^{\circ} \mathrm{C}$ is appropriate.

The influence of polymerization time on the product scale inhibition rate. Maleic anhydride was $9.8 \mathrm{~g}, 50 \%$ sodium hydroxide solution adjust $\mathrm{pH}$, hydrolysis $\mathrm{pH}$ value $6 \sim 7$, cyclization temperature was $60{ }^{\circ} \mathrm{C}$, cyclization time $2 \mathrm{~h}$, cyclization $\mathrm{pH}$ was 4 , catalyst was $0.33 \mathrm{~g}$, initiator dosage was $0.5 \mathrm{~g}$, polymerization temperature was $82^{\circ} \mathrm{C}$, poly epoxy succinic acid dosage was $8 \mathrm{mg} / \mathrm{L}$. The polymerization time effect on the product of $\mathrm{BaSO}_{4}$ scale inhibition performance, see Fig.5. 


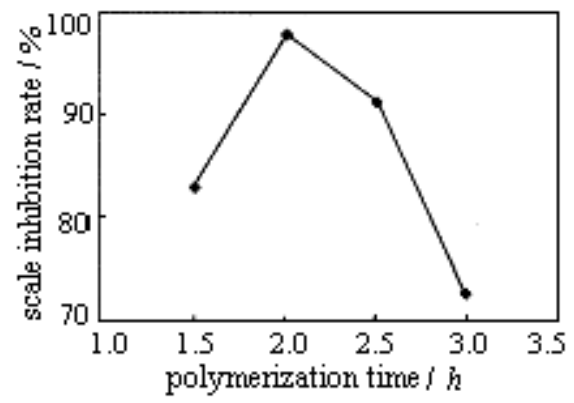

Fig. 5 The influence of polymerization time on the product scale inhibition rate

Time is too short, the product of polymerization degree is too small; the time is too long, polymerization of the products through the large. These two conditions will affect the product of relative molecular weight, thus affecting the scale inhibition effect. We can see from Fig.5, the polymerization time $2 \mathrm{~h}$ is appropriate.

To sum up, the best technology for maleic anhydride was $9.8 \mathrm{~g}, 50 \%$ sodium hydroxide solution adjust $\mathrm{pH}$, hydrolysis $\mathrm{pH}$ was $6 \sim 7$, catalyst was $0.33 \mathrm{~g}$, cyclization temperature was $60^{\circ} \mathrm{C}$, cyclization $\mathrm{pH}$ was 4 , cyclization time was $2 \mathrm{~h}$, initiator dosage was $0.5 \mathrm{~g}$, polymerization time was $2 \mathrm{~h}$, polymerization temperature was $82^{\circ} \mathrm{C}$.

Scale inhibition performance evaluation. According to the oil and gas industry standards SY/T 5673-93 " Anti scaling agent performance evaluation method for oilfield ", scale inhibition performance of synthetic product, scale object for barium sulfate and strontium sulfate. barium, strontium, which ion concentrations were determined by atomic absorption spectrophotometer, the working parameters see Table 1.

Table 1 The operation parameters of atomic absorption spectrophotometer

\begin{tabular}{cccc}
\hline Determination & Light source & Wavelength $(\mathrm{nm})$ & Flame \\
\hline $\mathrm{Ba}$ & $\mathrm{Ba}$ cathode lamp & 553.5 & Acetylene - air \\
$\mathrm{Sr}$ & Sr cathode lamp & 461.5 & Acetylene - air \\
\hline
\end{tabular}

In the following conditions of synthetic products were determined, hydrolysis $\mathrm{pH}$ was 6 , the amount of catalyst was $0.33 \mathrm{~g}$, hydrogen peroxide dosage was $12 \mathrm{~mL}$, cyclization $\mathrm{pH}$ was 4 , cyclization temperature was $60^{\circ} \mathrm{C}$, cyclization time was $2 \mathrm{~h}$, initiator dosage was $0.5 \mathrm{~g}$, polymerization temperature was $82^{\circ} \mathrm{C}$, polymerization time was $2 \mathrm{~h}$. The poly epoxy succinic acid dosage on the effect of scale inhibition rate is shown in Table 2, Table 3.

Table 2 The relationship between dosage of poly epoxy succinic acid and scale inhibition rate (barium sulfate)

\begin{tabular}{cc}
\hline Poly epoxy succinic acid amount $\left(\mathrm{mg} . \mathrm{L}^{-1}\right)$ & Scale inhibition rate $(\%)$ \\
\hline 2 & 7.1 \\
5 & 35.6 \\
8 & 98.0 \\
15 & 100 \\
20 & 100 \\
\hline
\end{tabular}

We can see from table 2, when poly epoxy succinic acid added $8 \mathrm{mg} / \mathrm{L}$, the barium sulfate scale inhibition rate can reach $98 \%$; the adding amount $15 \mathrm{mg} / \mathrm{L}$, scale inhibition rate reached $100 \%$. In view of this, poly epoxy succinic acid has good scale inhibition effect on barium sulfate.

Table 3 The relationship between dosage of poly epoxy succinic acid and scale inhibition rate (strontium sulfate)

\begin{tabular}{|c|c|}
\hline Poly epoxy succinic acid amount $\left(\mathrm{mg} . \mathrm{L}^{-1}\right)$ & Scale inhibition rate $(\%)$ \\
\hline 10 & 30.5 \\
\hline 20 & 55.9 \\
\hline 30 & 81.1 \\
\hline 40 & 90.4 \\
\hline 50 & 98.0 \\
\hline
\end{tabular}


We can see from table 3, the poly epoxy succinic acid synthesis in this experiment, the effect of strontium sulfate scale inhibition than barium sulfate scale inhibition effect slightly worse. Because in the experimental result and the evaluation criteria the solution configuration method has relates greatly, dosage relations may make the performance of scale inhibition of poly epoxy succinic acid is restricted to a certain extent.

\section{Conclusions}

Using maleic anhydride as raw material, sodium tungstate as catalyst, hydrogen peroxide as oxidizing agent, calcium hydroxide as initiator, the synthesis conditions of the best scale inhibition performance of polyepoxy succinic acid were studied by using the method of polymerization: maleic anhydride dosage was $9.8 \mathrm{~g}$, hydrolysis $\mathrm{pH}$ value $6 \sim 7$, the catalyst sodium tungstate amount used was $0.33 \mathrm{~g}$, the cyclization response $\mathrm{pH}$ value was 4 , the initiator calcium hydroxide joins the quantity was $0.5 \mathrm{~g}$, polymerization temperature was $82^{\circ} \mathrm{C}$, polymerization time was $2 \mathrm{~h}$.

The scale inhibition performance of the epoxy succinic acid was evaluated by atomic absorption spectrophotometry: When gathers the epoxy succinic acid joins the quantity is $8 \mathrm{mg} / \mathrm{L}$, the best effect of the scale inhibition rate of barium sulfate was $98 \%$; When gathers the epoxy succinic acid joins the quantity was $40 \mathrm{mg} / \mathrm{L}$, the best effect of the scale inhibition rate of strontium sulfate was $90.4 \%$. The results prove that poly epoxy succinic acid has a unique scale inhibition effect on barium sulfate scale, and the amount of use is lower.

\section{References}

[1] Li Dan, Zhang Puyu, Reach on Synthesis of Methods Polyepoxysuccinic Acid. Guangdong Chemical Industry. 4(2012)91-92

[2] Yu Yuxin, Li Jianjun, Study on poly epoxy succinic acid disodium salt calcium carbonate scale inhibiting performance. Environmental Engineering. 1 (2005)85-87.

[3] Bush R D, Heinzman S W., U.S. Patent 4,654,159.(1987)

[4] Brown J M, McDowell J F, Chang K T., U.S. Patent 5,062,962.(1991)

[5] McGiffney G J., U.S. Patent 5,866,011.(1999)

[6] Chen Yanmin, Sun Caixia, Xu Huiwu. Performance of Biode gradation of Environment-friendly Corrosion and Scale Inhibitor. HENAN SCIENCE, 9(2014)1842-1844.

[7] Hu Xinggang, Jin Xiaoxia, Sun Ji. Researches on the application of the "friendly-to-environment" type scale inhibiting disperser and its evaluation. Industrial Water Treatment. 11(2003)30-34.

[8] Zhang Lihua, Zheng Chengsong, Zhang yan, elt. Development of environment friendly polymer PESA. Industrial Water Treatment. 5(2010)5-8.

[9] Yang Yingqin, Duan Xiangzhi, Ping Kun. Synthetic condition of biodegradable PESA and its scale inhibiting properties. Journal of Xinyang Normal University. 2(2004)221-223.

[10] Zhang Sufang, Shu Yuanjie, Research on Performance of Calcium Scale Inhibition by Modifidied Polyepoxysuccinic Acid. CHINA WATER \& WASTEWATER. 21(2008)42-44. 